\title{
BLOQUEOS DE PARED TORÁCICA BAJO VISIÓN ECOGRÁFICA DIRECTA EN CIRUGÍA DE MAMA
}

\author{
María Loreto Astudillo A. y Daniella Rigo-Righi I. ${ }^{1}$
}

Key words: Pectoral block, serratus block, ultrasound block.

\section{INTRODUCCIÓN}

El manejo del dolor postoperatorio luego de una intervención quirúrgica constituye uno de los pilares más importantes del acto anestésico. La cirugía de mama actualmente es muy frecuente en nuestros hospitales y clínicas. Por pequeña que sea la cirugía realizada, se asocia a dolor postoperatorio significativo con gran variabilidad entre pacientes y a largo plazo puede llegar a producir dolor postoperatorio prolongado ${ }^{1,2}$; por lo tanto, su manejo en el postoperatorio inmediato adquiere gran relevancia. Actualmente, las opciones analgésicas para cirugías de mama (mastectomías totales o parciales, implantes mamarios, reducciones o reconstrucciones mamarias) pueden ser sistémicas o regionales.

Las técnicas regionales más conocidas y consideradas como gold estándar han sido la analgesia peridural torácica y el bloqueo paravertebral. Desde la década de los 90 se describe la utilidad del bloqueo paravertebral en cirugía de mama; incluso como técnica anestésica. Pero éste no es capaz de entregar un bloqueo sensitivo completo de la cara anterior del tórax, porque la inervación no es exclusiva de los nervios espinales; ya que también participan ramas del plexo braquial. Además, hay que considerar sus posibles complicaciones, tales como punción vascular, neumotórax y difusión peridural e intratecal ${ }^{3}$. Otro inconveniente es que se debe realizar con el paciente despierto para mantener una posición adecuada y requiere más de una punción; lo que produce disconfort y mayor ansiedad ${ }^{4,5,6}$.

Por otro lado, la analgesia peridural torácica es una técnica que necesita experiencia del operador, y muchas veces la complejidad y los riesgos del procedimiento son de un riesgo/beneficio que debe ser analizado en cada enfermo. Otras opciones, como la infiltración de la herida operatoria, serían insuficientes en el control óptimo del dolor.

Los bloqueos nerviosos periféricos bajo visión ecográfica directa se han tornado fáciles y seguros. Los equipos disponibles permiten una visualización precisa de las estructuras; esto, asociado al diseño de las agujas ecorrefringentes han reducido las complicaciones y aumentado la tasa de éxito, permitiendo un aumento en el interés por su realización y el desarrollo de nuevas técnicas.

La presente revisión busca describir en forma detallada la anatomía, las técnicas ecográficas descritas en los últimos años y nuestra experiencia clínica sobre bloqueos de la pared torácica bajo visión ecográfica para manejo del dolor.

\section{Anatomía}

Para comprender estas técnicas, primero se debe conocer la anatomía de la zona. La pared torácica posee 2 grupos de músculos.

Músculos intrínsecos: intercostales y diafragma, actúan sobre las costillas para modificar el volumen de la cavidad torácica.

Músculos extrínsecos: pectoral mayor y menor, serrato anterior y dorsal ancho, que se insertan en el tórax, pero tienen por función movilizar el miembro superior (Figura 1).

En relación a la inervación, existen 3 grandes grupos de nervios (Figura 2):

Un primer grupo de nervios que proviene del plexo braquial, inervan los músculos más anterio-

Departamento de Anestesiología y Reanimación, Hospital Fuerza Aérea de Chile y Fundación Arturo López Pérez.

Profesor Asociado Universidad de Valparaíso. 


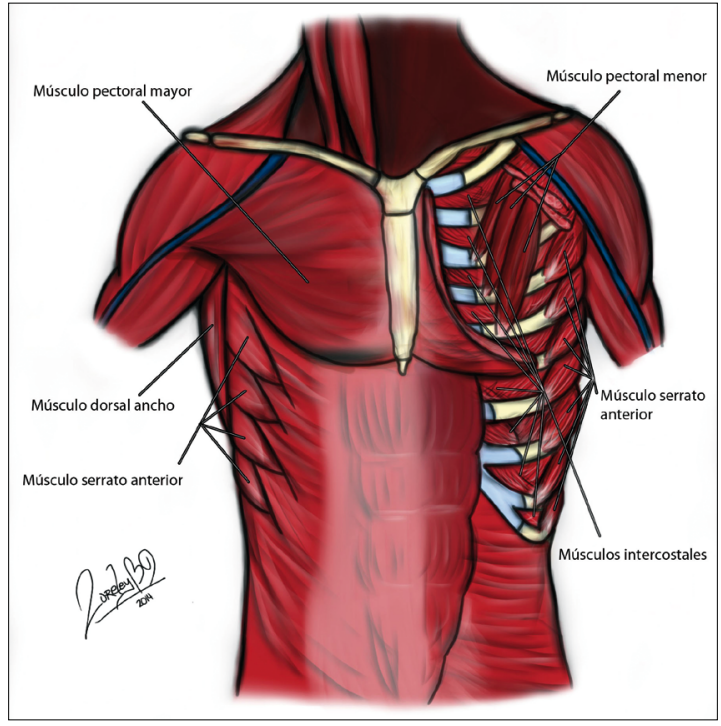

Figura 1. Músculos cara anterior del tórax.

res de la pared torácica; los músculos pectorales. El nervio pectoral lateral (C5 - C7), es el más grande, desciende hacia abajo y adentro y se divide en una rama que cruza oblicuamente la cara anterior de la arteria axilar, atraviesa la aponeurosis clavipectoral y aborda la cara profunda del músculo pectoral mayor; la otra rama se une al nervio pectoral medial formando el asa de los pectorales e intercambiando fibras nerviosas con este nervio.

El nervio pectoral medial (C8 y T1) surge del cordón medial por detrás de la clavícula, desciende hacia abajo y adelante y se divide en una rama para el pectoral menor y otra para formar el asa de los pectorales. De la convexidad del asa se desprenden unos ramos nerviosos que cruzan el borde superior del pectoral menor y se distribuyen en el músculo pectoral mayor, mientras otros alcanzan el pectoral menor y lo inervan ${ }^{5}$.

El segundo grupo proviene de las divisiones anteriores de nervios intercostales torácicos (T2 T6). Desde la espalda al esternón viajan entre los músculos intercostales. Su rama lateral inerva al músculo serrato e intercostal externo hasta línea axilar media. Mientras que la rama anterior, cruza la arteria mamaria interna, perfora los músculos intercostal interno y pectoral menor para inervar la zona medial de la mama. A nivel de T2, no se divide y se llama nervio intercostobraquial.

El último grupo inerva los músculos más laterales y también nacen en el plexo braquial. El nervio torácico largo o nervio serrato anterior (C5 - C7), que al dañarse durante la disección axilar produce escápula alada; y el nervio toracodorsal, que inerva

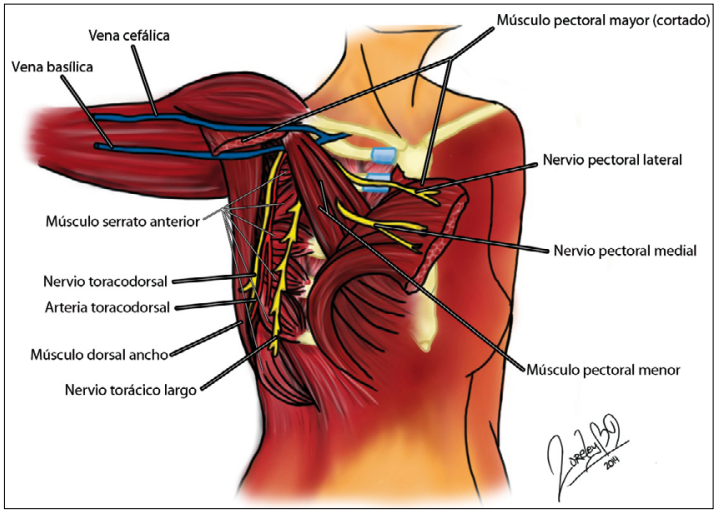

Figura 2. Inervación cara anterior del tórax.

el dorsal ancho y la cara posterior de axila.

La inervación de la glándula mamaria está dispuesta de forma segmentaria, y proviene de las ramas cutáneo anterior y laterales de los nervios intercostales $4^{\circ}$ a $6^{\circ}$. Cranealmente participan las ramas del plexo cervical superficial con los nervios supraclaviculares. Las ramas cutáneas laterales del $2^{\circ}$ al $6^{\circ}$ nervio intercostal transitan a través de los músculos intercostales y a nivel de la línea medio axilar, pasan a través del músculo serrato anterior y se dividen en dos ramas una anterior y otra posterior. Las ramas anteriores corren a lo largo de la fascia pectoral y perforan el borde lateral del músculo pectoral mayor antes de entrar en el parénquima de la mama. La cola axilar de la mama está inervada por ramas cutáneas laterales de los nervios intercostales del $1^{\circ}$ al $4^{\circ}$ y las ramas cutáneas del nervio intercostobraquial ${ }^{7}$.

Los nervios intercostales conducen fibras sensitivas para la piel mamaria, fibras simpáticas para los vasos sanguíneos y el músculo liso de la piel suprayacente y del pezón. La rama cutánea del $4^{\circ}$ nervio intercostal es reconocida como la rama más importante en la sensibilidad del pezón ${ }^{6}$.

\section{Descripción de bloqueos}

Actualmente, podemos encontrar en la literatura varios bloqueos de la pared torácica guiados con ecografía. Todos depositan el anestésico local en planos interfasciales, no producen bloqueo simpático y no necesitan opioides. Además son superficiales, rápidos y fáciles de realizar².

El grupo de Blanco y col. ha descrito 3 bloqueos: el bloqueo pectoral I (Pecs I), el bloqueo pectoral modificado (Pecs II) y el bloqueo del plano serrato anterior (SAP block). 
Fajardo y col. ${ }^{7,10}$ realizan 3 bloqueos en cirugía de mama ambulatoria dirigidos a interrumpir el impulso nervioso de las ramas cutáneas intercostales.

Técnicamente son muy similares, salvo pequeñas variaciones sonográficas. Para poder estudiarlas con mayor facilidad, las podemos clasificar según la ubicación del transductor en anteriores y laterales.

Los bloqueos anteriores van dirigidos a bloquear los nervios pectorales y/o los nervios intercostales. Mientras que los laterales bloquean nervios intercostales, el nervio torácico largo y el toracodorsal.

\section{Bloqueos anteriores}

\section{Bloqueo pectoral I}

El objetivo es depositar el anestésico local en el plano interfascial entre la hoja profunda de la fascia pectoral y la fascia clavipectoral; es decir, entre los músculos pectoral mayor y menor. Permite el bloqueo sensitivo de ambos músculos. El paciente se ubica en decúbito supino con el brazo ipsilateral pegado al cuerpo y la cabeza girada hacia el lado contrario. Existen 2 abordajes:

Abordaje coracoideo: el transductor se ubica bajo la clavícula, medial a la apófisis coracoides, similar a la posición para bloqueo infraclavicular. Bajo el tejido subcutáneo se observan el músculo pectoral mayor, el pectoral menor y profundidad, se observa la vena y arteria axilar.

Se introduce la aguja por el borde superior de la sonda en un ángulo de $45^{\circ}$, en dirección a la línea hiperecogénica entre ambos músculos pectorales ${ }^{8}$. Este abordaje tiene el inconveniente del contacto óseo que puede dificultar la movilización de la sonda y disminuir la posibilidad de maniobrar con la aguja $^{9}$ (Figura 3).

Abordaje infraclavicular: Pérez y col. describen un acceso diferente. Se ubica el transductor bajo el tercio externo de la clavícula, transversal al eje del paciente. Se identifican los músculos pectoral mayor y menor; con Doppler se ubica la arteria toracoacromial y la vena cefálica. En un plano más profundo se visualizan las costillas y la pleura.

La aguja se introduce desde medial a lateral en un ángulo de $45^{\circ}$, alejándose lo más posible de estructuras como la pleura y vasos sanguíneos. El nervio pectoral lateral se encuentra lateral a la arteria. La hoja posterior se despega fácilmente con la infiltración del fluido. Es en este plano donde se logra el bloqueo de los nervios pectorales medial y lateral, pues ambos atraviesan la hoja profunda de la fascia pectoral para inervar al músculo pectoral mayor $^{10}$ (Figura 4).

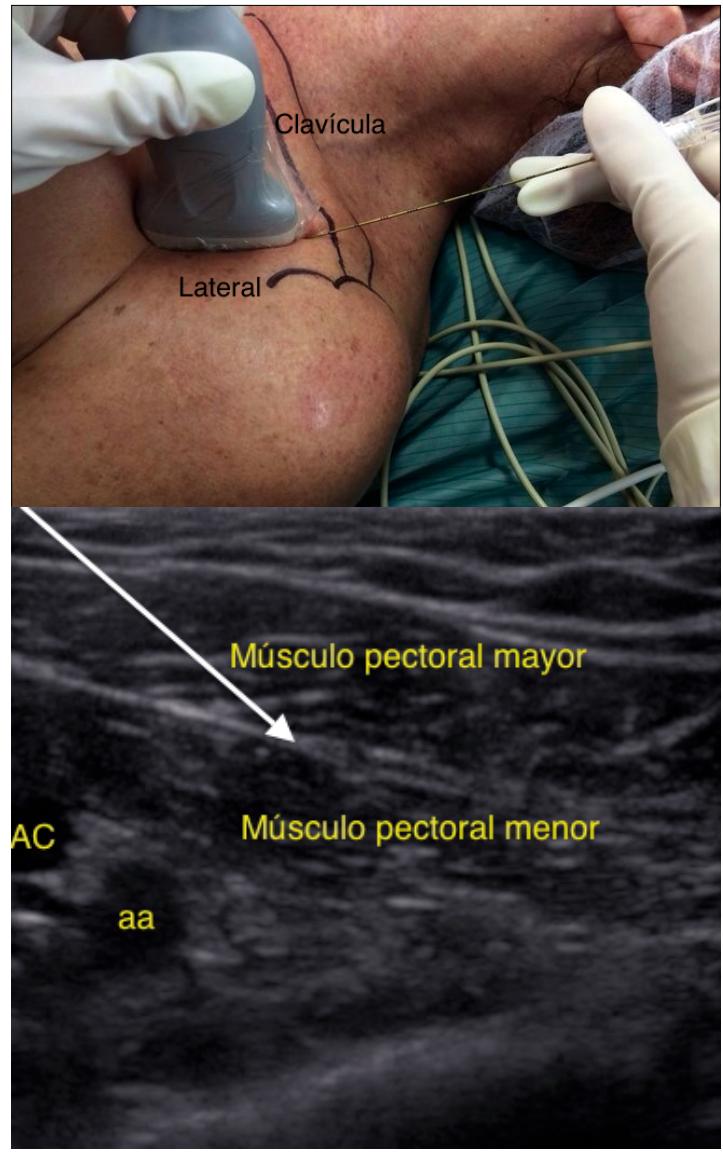

Figura 3 Posición transductor y ecografía del bloqueo pectoral I, abordaje coracoideo. Flecha indica fascia clavipectoral.

Este grupo ha usado este bloqueo en pequeñas y grandes cirugías de mama, consiguiendo una reducción de los requerimientos de analgesia sistémica en el perioperatorio y mejorando la satisfacción de los pacientes. Este acceso tiene por ventaja la menor probabilidad de punción de vasos y pleura por su orientación de medial a lateral. Además, la inserción de la aguja no encuentra obstáculos óseos y sería más fácil la instalación de catéteres ${ }^{11}$.

La simple inyección en el plano interfascial de los pectorales no es suficiente para alcanzar las ramas anteriores de los nervios intercostales. Por esta razón se creó el Pecs I modificado o Pecs II².

\section{Bloqueo pectoral II}

Este bloqueo pretende producir un bloqueo a nivel axilar mediante dos punciones. La primera, corresponde a un bloqueo Pecs I y luego, otra punción que se realiza entre el pectoral menor y el músculo serrato. La sonda se ubica bajo el tercio lateral de 


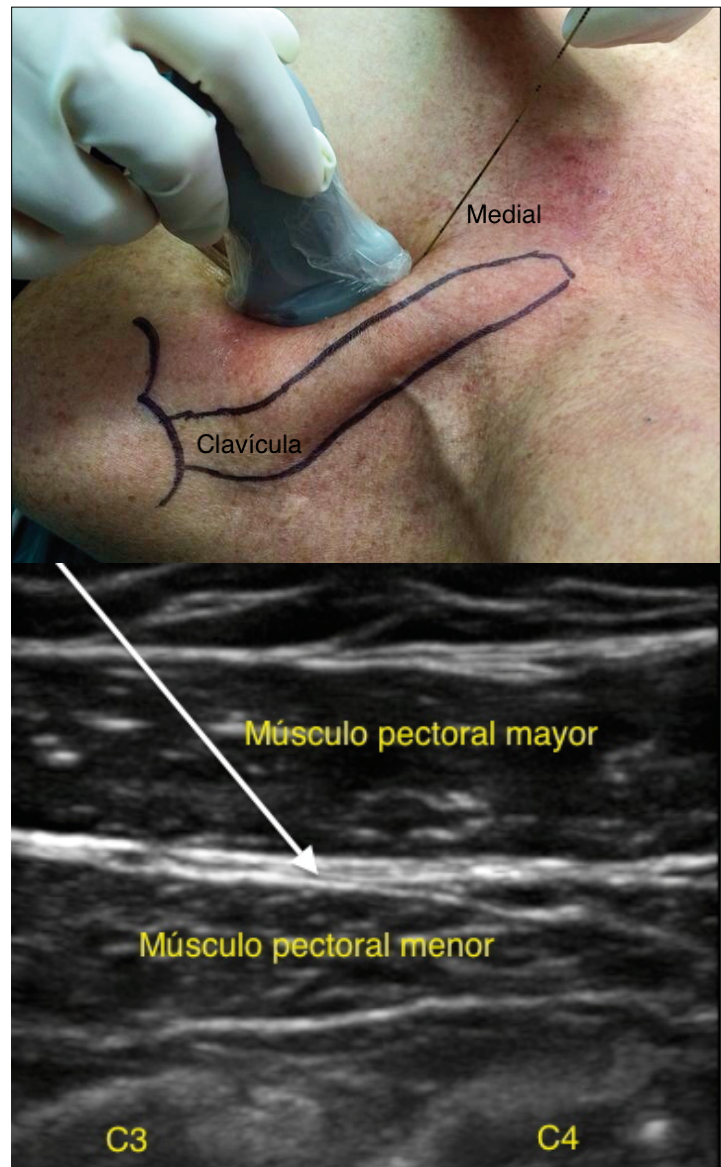

Figura 4. Posición y sonoanatomía bloqueo pectoral I, abordaje infraclavicular. Flecha indica lugar de depósito de anestésico local.

la clavícula. Se visualizan el músculo subclavio, la arteria y la vena axilar, y luego se desplaza hacia la axila, hasta identificar el músculo pectoral menor. Se cuentan las costillas moviéndose hacia distal y lateral hasta alcanzar el borde lateral del pectoral menor. Sobre la tercera costilla podemos ver la continuación del ligamento de Gerdy y bajo él otro músculo cubriendo las costillas 2,3 y 4 ; el serrato anterior. Este es el punto de entrada y se deposita la solución entre los músculos pectoral menor y serrato ${ }^{2}$ (Figura 5).

Es un abordaje que permite bloquear los nervios pectorales, nervios intercostobraquial, intercostales 3 - 6 y el nervio torácico largo. Muy útil para el manejo del dolor en cirugías que utilizan prótesis o expansores subpectorales.

Bloqueo pectoral con punción única: Desroches et al. mediante la disección en cadáveres ha

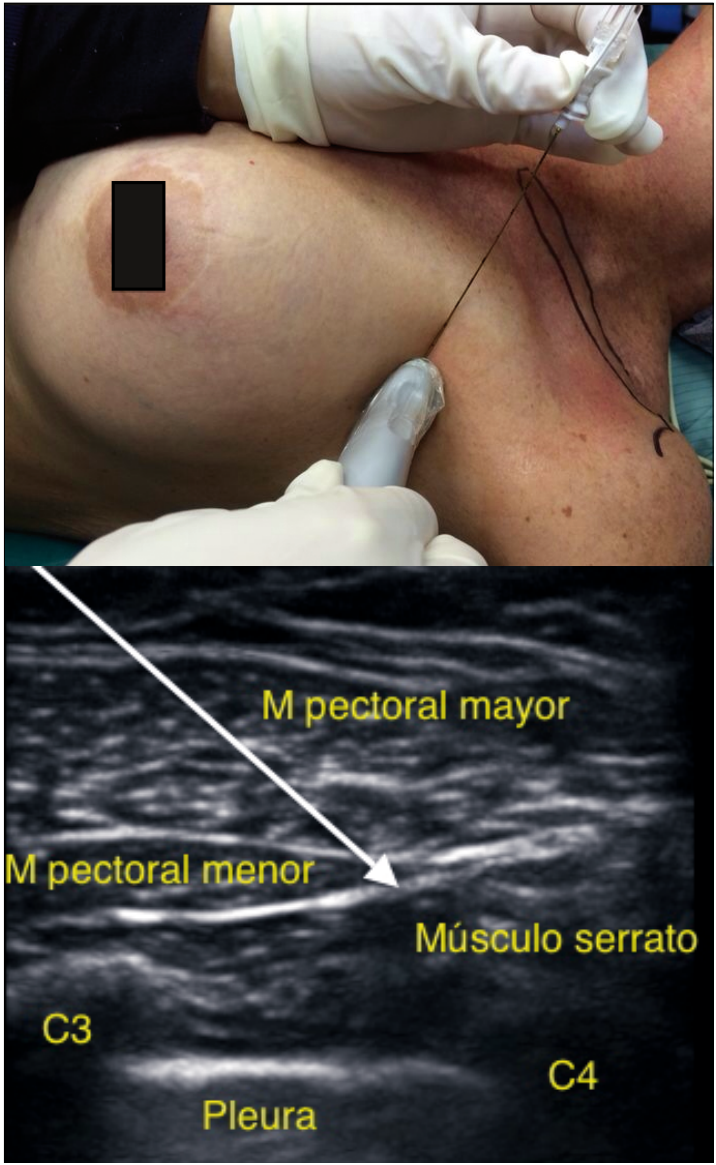

Figura 5. Ubicación de transductor y sonoanatomía del bloqueo pectoral II. Flecha indica zona donde se deposita anestésico local.

demostrado la posibilidad de bloquear bajo visión ecográfica los nervios pectorales con una sola punción. Ubicando el transductor $4 \mathrm{~cm}$ caudal a la apófisis coracoides con su margen medial en línea media clavicular, se inserta la aguja in plane en dirección medial a lateral, perpendicular al músculo pectoral menor. Se deposita la solución en 3 sitios. Primero, en la zona profunda y lateral del pectoral menor; luego, retirando la aguja hasta que la punta se ubica en el segundo sitio, entre ambos pectorales. Por último, dirigiéndose hacia la superficie, se infiltra en la fascia posterior del músculo pectoral mayor (Figura 6). Además, la tinción del sitio de punción con azul de metileno demuestra que no hay ascenso de solución hacia el plexo braquial ${ }^{12}$.

Bloqueo de ramas cutáneas: El bloqueo de los nervios pectorales en dosis única ofrece buena analgesia y ventajas respecto a otras técnicas anal- 


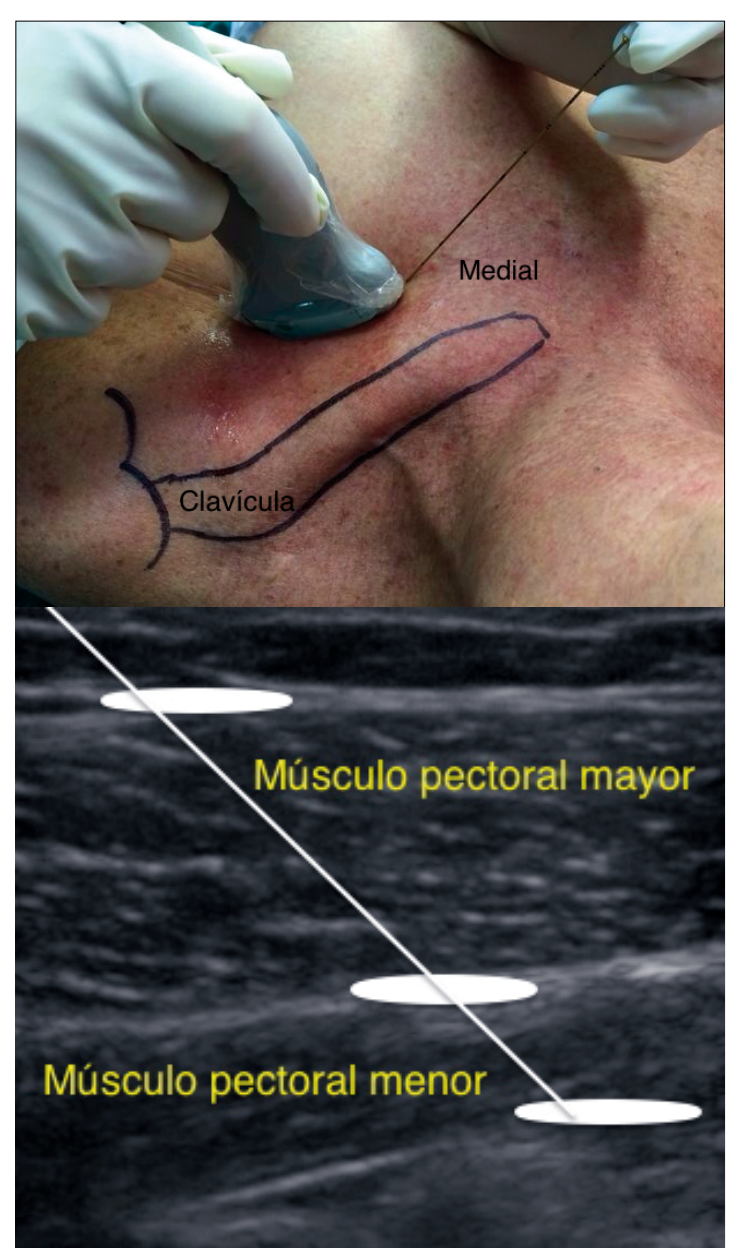

Figura 6. Ubicación de sonda y sonoanatomía bloqueo pectoral con punción única. Semicírculos marcan sitios de depósito de anestésico local.

gésicas en cirugía de mama con implantes. No obstante, su eficacia es limitada ya que no proporciona analgesia adecuada en la zona del complejo areolapezón, al ser la inervación de esta zona dependiente de los nervios intercostales. Una alternativa sugerida por el grupo de Fajardo y col, es el bloqueo eco guiado de las ramas cutáneas anteriores (BRCA) y laterales (BRCL) de los nervios intercostales en la región anterolateral del tórax ${ }^{7}$.

En el BRCL se ubica el transductor en tercio externo de la clavícula a nivel del segundo espacio intercostal, ligeramente oblicuo al cuerpo (Figura 7).

Se ubican las mismas estructuras que al realizar un bloqueo pectoral II. Se observa tejido subcutáneo, el músculo pectoral mayor, menor y serrato, y por último, los músculos intercostales. Destaca que el sitio donde se ubica la punta de la aguja es entre los músculos serrato e intercostales. Se desplaza la

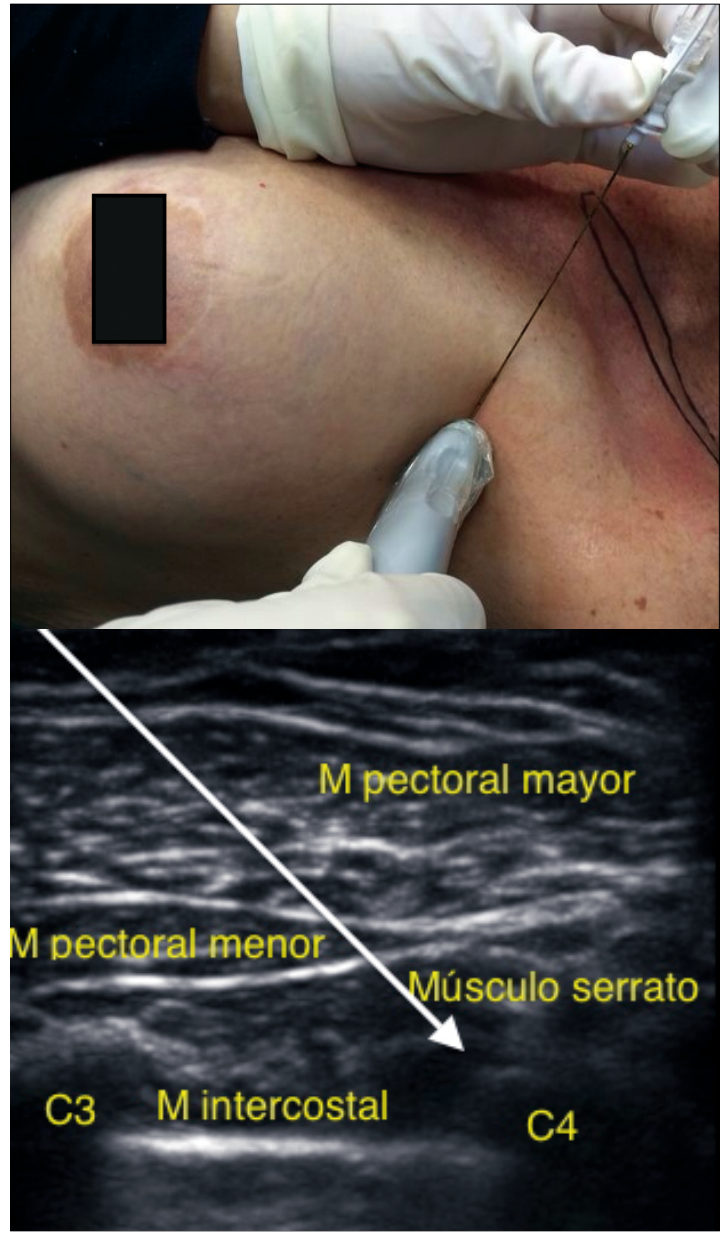

Figura 7. Sonoanatomía BRCL. Flecha entre músculos serrato e intercostal donde se deposita anestésico local.

sonda hacia caudal para bloquear las ramas de espacios $3^{\circ}$ a $5^{\circ}$.

En el BRCA la sonda se coloca en la línea paraesternal. Se observa músculo pectoral mayor y luego los intercostales, pleura y pulmón. La aguja ingresa desde el borde caudal hasta el plano interfascial entre los músculos pectoral e intercostales (Figura 8). Se administra $3 \mathrm{ml}$ de anestésico local por nivel. Se debe utilizar doppler para identificar la arteria torácica interna y sus ramas mamarias.

En un grupo de 44 pacientes sometidas a cirugía de mama sin implantes utilizando el BRCL combinado con BRCA, Fajardo y col. demostraron una efectividad analgésica de $95 \%$ con una duración de 18 a 24 horas, sin necesidad de analgesia de rescate en domicilio y una excelente satisfacción usuaria. Mediante evaluación clínica, imagenológica y en cadáveres, vieron la distribución del anestésico lo- 


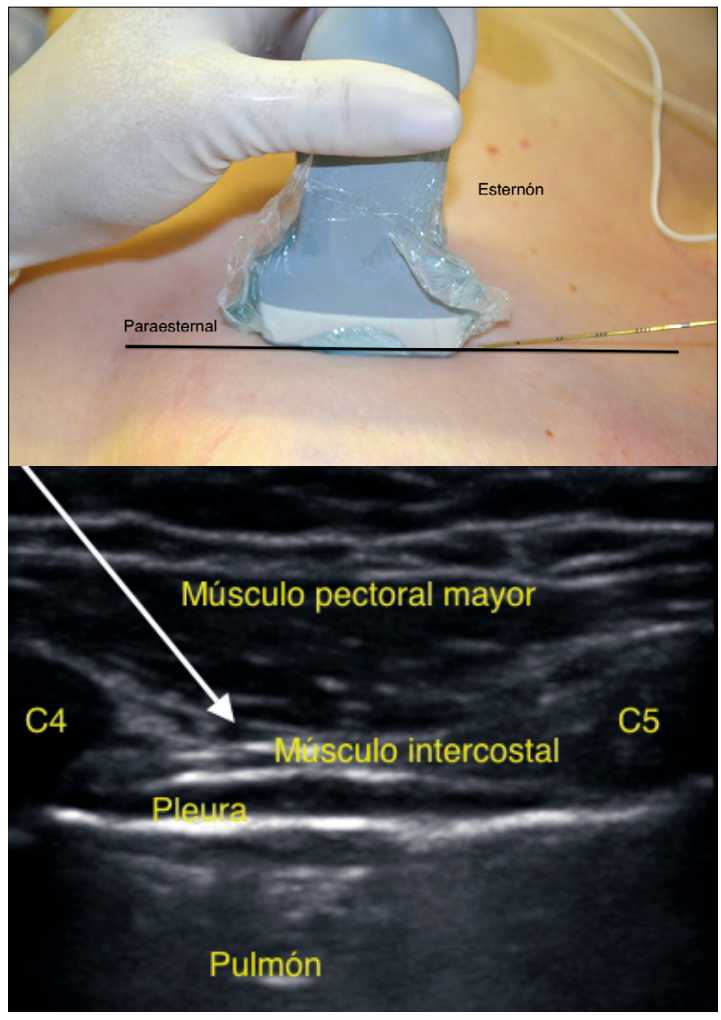

Figura 8. Ubicación de transductor y sonoanatomía de BRCA. Flecha marca sitio donde se deposita el anestésico local.

cal; éste se desplazó hasta línea axilar posterior en el primer bloqueo y hasta 5 centímetros hacia lateral en la punción paraesternal; bañando las ramas intercostales involucradas en la sensibilidad ${ }^{7}$.

\section{Bloqueos laterales}

Bloqueo del plano serrato anterior: El bloqueo del plano serrato es una técnica reciente, poco estudiada y fácil de realizar con bajo potencial de efectos adversos asociados a la inyección cercana a estructuras vasculares. No requiere múltiples punciones ni cambios de orientación de la aguja. Otorga analgesia de las paredes anterior y lateral del tórax, no describiéndose hasta ahora ninguna complicación.

La aguja se inserta a nivel de la quinta costilla en el punto que intersecta con la línea axilar media (Figura 9). Se identifican desde superficial a profundo el músculo dorsal ancho, el serrato anterior y los músculos intercostales. En base a estudios sonográficos, el anestésico local podría depositarse en dos espacios virtuales: uno superficial entre el músculo dorsal ancho y serrato (Figura 10); y otro

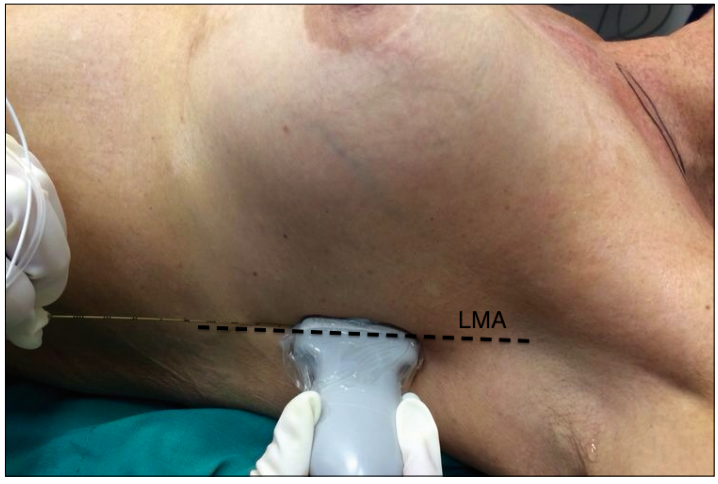

Figura 9. Ubicación de sonda para realizar bloqueo del plano serrato (SAP). LMA: línea axilar media.

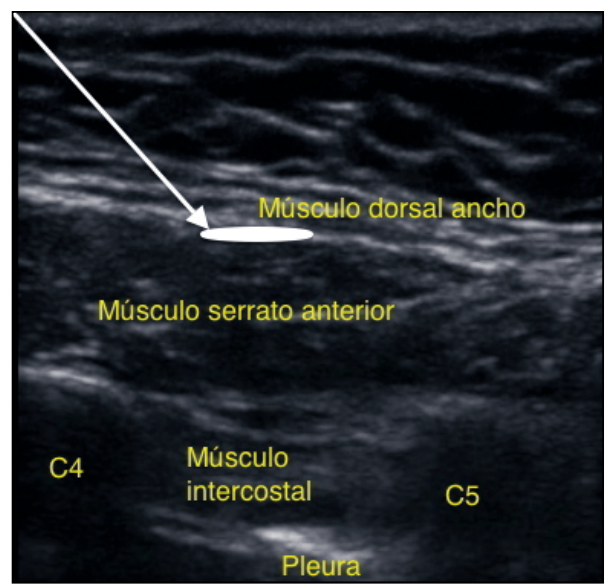

Figura 10. Sonoanatomía de SAP superficial.

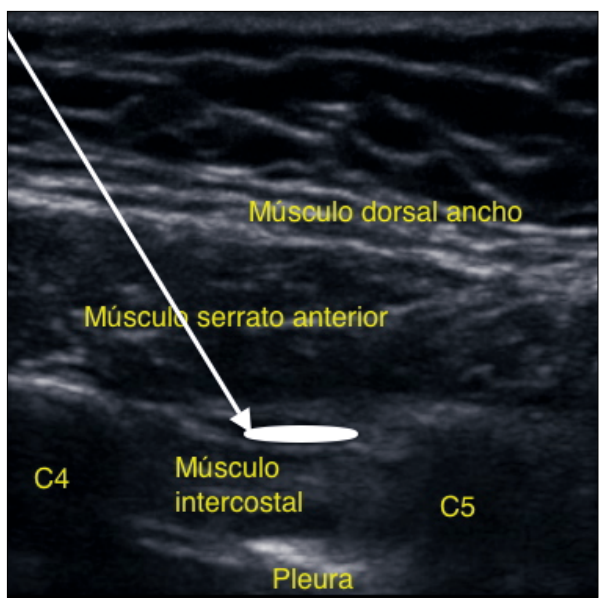

Figura 11. Sonoanatomía SAP profundo. 
profundo, bajo el músculo serrato (Figura 11).

Blanco y col. ${ }^{13}$ compararon ambos sitios de depósito en 4 pacientes despiertas. Bajo visión ecográfica directa inyectaron una mezcla de $0,4 \mathrm{ml} /$ $\mathrm{kg}$ de levobupivacaína $0,125 \%$ con gadolinio 0,1 $\mathrm{mmol} / \mathrm{kg}$. Realizaron 2 bloqueos en cada paciente: uno de ellos superficial al músculo serrato anterior y al otro lado, fue profundo, bajo el serrato. A los 30 minutos, delinearon el área sin sensibilidad usando una aguja hipodérmica. Luego de la infiltración, las 4 pacientes voluntarias presentaron parestesias entre los dermatomas T2 a T9, parálisis y disminución en aducción cruzada de la extremidad, bilaterales.

En el caso de la infiltración superficial, la duración promedio de las parestesias por efecto sobre los nervios intercostales fue 752 minutos y 778 minutos para el bloqueo motor del nervio torácico largo. Luego de la infiltración profunda, la duración de las parestesias fue 386 minutos y el bloqueo motor duró 502 minutos.

La RNM con gadolinio mostró una mejor extensión del bloqueo realizado a nivel superficial; ya que se visualiza la banda de señal de alta intensidad en la zona anteroposterior y a lo largo del hemitórax respectivo ${ }^{13}$.

El grupo de Blanco concluyó que el bloqueo superficial es más efectivo que el profundo. Sin embargo, el estudio que realizaron fue sólo descriptivo y se requieren estudios controlados y randomizados que midan efectivamente la utilidad de este bloqueo.

\section{Otras utilidades de los bloqueos de pared torácica}

Los bloqueos de pared torácica bajo ecografía no sólo se han descrito para cirugía de mama sino también para trauma torácico, fracturas costales, drenajes pleurales ${ }^{15}$, por lo que sus utilidades se extenderían más allá de la cirugía de mama.

\section{Experiencia}

En la actualidad en nuestro centro, el grupo de anestesiólogos está utilizando el bloqueo a nivel del plano serrato anterior en cirugía de mama.

Desde febrero a la fecha contamos con más de 80 procedimientos; realizamos el bloqueo en pacientes dormidas antes del inicio de la cirugía, administramos $20 \mathrm{ml}$ de una solución de levobupivacaína $0,25 \%$. En estas pacientes hemos alcanzado una analgesia adecuada en la mayoría de los casos, sin complicaciones.
No sólo lo estamos utilizando para cirugía de mama sino también tenemos casos de pacientes sometidos a videotoracoscopía e instalación de drenajes pleurales, donde hemos realizado bloqueo del plano serrato con EVA postoperatorio menor a $4 \mathrm{y}$ sin consumo de opioides de rescate durante las primeras 24 horas.

\section{CONCLUSIONES}

El uso de técnicas regionales bajo visión ecográfica no sólo ha permitido masificar y realizar en forma más segura los bloqueos regionales; sino que además, ha incrementado la búsqueda de nuevos accesos y técnicas que amplían nuestros horizontes y mejoran la calidad de la atención a nuestros pacientes.

El grupo de Blanco ha descrito varias técnicas que permiten bloquear una zona olvidada por muchos años; donde la analgesia peridural y paravertebral ofrecen una lista innegable de beneficios. Los bloqueos interfasciales a nivel del tórax son una alternativa válida en ciertas cirugías. Tienen características únicas: se pueden realizar con el paciente dormido, se pueden visualizar las estructuras y el sitio donde se deposita el anestésico local, son fáciles de realizar y tienen pocas complicaciones.

La analgesia/anestesia en cirugía de mama dependerá de la extensión de la cirugía. Para la inserción de prótesis subpectorales, el tejido dañado se limita al músculo pectoral mayor y al serrato por lo que requerirá el bloqueo de los nervios pectoral lateral y medial. Para procedimientos más extensos como tumorectomías, mastectomías o ganglio centinela axilar será necesario bloquear los nervios intercostales. Para reconstrucciones con músculo dorsal ancho, el bloqueo debe incluir los nervios torácico largo y toracodorsal.

Por anatomía, la fascia que rodea al músculo serrato, se continúa con la fascia clavipectoral y con la fascia del dorsal ancho; al administrar una solución a nivel del plano del músculo serrato, ésta difundiría entre los músculos pectorales y entre músculos serrato y dorsal ancho, bloqueando los nervios pectorales hacia anterior y los nervios toracodorsal y torácico largo hacia lateral. La difusión del anestésico local hacia planos más profundos bloquearía los nervios intercostales. Por lo tanto, sería el bloqueo más completo para cualquier tipo de cirugía de mama. Faltan trabajos clínicos que demuestren esta teoría, por ahora sólo contamos con series de casos.

Revisada la anatomía y descritas las técnicas 
disponibles para lograr un bloqueo adecuado de la pared torácica; no nos queda más que animarnos a realizarlos en nuestra actividad habitual.

En nuestro centro ya iniciamos un estudio clínico prospectivo, randomizado y ciego en el cual se ha realizado bloqueo del plano serrato anterior en todas las cirugías de mama, con el objeto de determinar la calidad de la analgesia postoperatoria y las posibles complicaciones del procedimiento. Pronto expondremos los resultados.

\section{REFERENCIAS}

1. Kaunisto MA, Jokela R, Tallgren M. Pain in 1,000 women treated for breast cancer: a prospective study of pain sensitivity and postoperative pain. Anesthesiology 2013; 119 1410-1421

2. Blanco R, Fajardo M, Parras Maldonado T. Ultrasound description of Pecs II (modified Pecs I): A novel approach approach to breast surgery. Rev Esp Anestesiol Reanim 2012; 59: 470-475.

3. Lönnqvist PA, MacKenzie J, Soni AK, et al. Paravertebral blockade: Failure rate and complications. Anesthesia 1995; 50: 813-815.

4. Ultrasound guided regional anesthesia. En: www. ultrasoundblock.com (Acceso el 7 de agosto de 2014).

5. Sopena-Zubiria LA. FernándezMeré. Bloqueo paravertebral torácico frente al bloqueo pararavertebral torácico más bloqueo de nervios pectorales en cirugía reconstructi- va de mama. Rev Esp Anestesiol Reanim 2012; 59: 12-17.

6. Wallaert M, Courivaud P, Mati $\mathrm{EH}$. Bloc interpectoral avec mise en place d'un cathéter pour analgésie postopératoire après chirurgie mammaire Catheter. Annales Francaises d'Anesthésie et de Réanimation (2014) article in press.

7. Fajardo Pérez M, García Miguel FJ. Analgesic combined lateral and anterior cutaneous branches of the intercostal nerves ultrasound block in ambulatory breast surgery. Cir May Amb 2012; 17: 95-104.

8. Blanco R. Correspondence. The 'pecs block': a novel technique for providing analgesia after breast surgery. Anaesthesia 2011; 66: 840852.

9. IX Curso de bloqueos nerviosos periféricos en anestesia regional y dolor. En: www.arydol.es (Acceso el 7 de agosto de 2014).

10. Fajardo Pérez M, Blanco Dávila R, García Miguel FJ. The usefulness of ultrasound-guided pectoral nerve block for postoperative pain control in reconstructive breast surgery. Rev Esp Anestesiol Reanim 2012; 59: 578-580.

11. Fajardo Pérez M, Miguel JG. A new approach to pectoralis block Anaesthesia 2013; 68: 422-437.

12. Desroches J, Grabs U, Grabs D. Selective Ultrasound Guided Pectoral Nerve Targeting in Breast Augmentation: How to Spare the Brachial Plexus Cords? Clinical Anatomy 2013; 26: 49-55.

13. Blanco R, Parras T, McDonnell JG. Serratus plane block: a novel ultrasound-guided thoracic wall nerve block. Anaesthesia 2013; 68: 1107-1113.

14. López-Matamala B, Fajardo M, Estébanez-Montiel B, et al. A new thoracic interfascial plane block as anesthesia for difficult weaning due to ribcage pain in critically ill patients. Med Intensiva 2013. http://dx.doi.org/10.1016/j.medin.2013.10.005

\section{Correspondencia a:}

Dra. María Loreto Astudillo Aliste mastudia@gmail.com 